\title{
Time taken from primary care referral to a specialist centre diagnosis of idiopathic pulmonary fibrosis: an opportunity to improve patient outcomes?
}

To the Editor:

The care of patients with idiopathic pulmonary fibrosis (IPF) has been transformed by the widespread approval of antifibrotic therapies [1]. Within primary care-based healthcare systems, the diagnosis of IPF and commencement of antifibrotic therapy typically requires a patient referral from a primary care physician to a respiratory physician in secondary care, with referral then made to a specialist interstitial lung disease (ILD) centre [2]. Following ILD centre review and multidisciplinary team (MDT) discussion, a diagnosis of IPF is made and antifibrotic therapy may be commenced.

To maximise the benefit of antifibrotic therapy, early diagnosis of IPF has been widely advocated [3-5]. However, while patient surveys and questionnaires consistently report significant delays to a definitive IPF diagnosis, understanding of any impact upon patient outcomes is limited [6]. The objective of this study was to investigate, within primary care healthcare systems, the time taken from the first primary care physician referral for symptom investigation to ILD specialist centre review and antifibrotic therapy commencement.

We identified patients with an MDT diagnosis of IPF between January 2012 and December 2017 in two specialist ILD clinics in two countries with a primary care healthcare system (University Hospital Southampton, Southampton, UK, and Mater Misericordiae University Hospital, Dublin, Ireland). The dates of primary care referral, first secondary care respiratory clinic contact, first ILD clinic review, antifibrotic commencement, antifibrotic discontinuation and date of death were determined through electronic case note review. Patients who were discussed in the MDT but not seen in the ILD clinic, or had insufficient clinical information, were excluded. The date of the census was April 2018. The study was approved by the London-Hampstead Research Ethics Committee (REC 17/LO/2037). Statistical analysis was conducted using SPSS version 25 (IBM, Armonk, NY, USA)

247 patients were identified (194 from Southampton and 53 from Dublin). 183 (74\%) were male and 162 (66\%) were ex-smokers. The median (interquartile range) time from primary care referral to secondary care respiratory clinic review was 47 (25-84) days, to ILD clinic 290 (133-773) days and to antifibrotic commencement 540 (282-1024) days.

We used Cox regression analysis to investigate for demographic variables associated with a $>12$ month period from general practitioner (GP) referral to ILD clinic. Male sex (hazard ratio (HR) 1.935, 95\% CI 1.141-3.281; $\mathrm{p}=0.014$ ), increasing body mass index (HR 1.081, 95\% CI 1.028-1.136; $\mathrm{p}=0.002$ ) and gastro-oesophageal reflux (HR 1.559, 95\% CI 1.010-2.405; $\mathrm{p}=0.045$ ) were significantly associated with a $>12$ month period from GP referral to ILD clinic. Age, smoking status, ILD centre and other comorbidities (COPD, diabetes and ischaemic heart disease) were not significantly associated.

Patients reviewed in the ILD clinic within 12 months of primary care referral $(\mathrm{n}=142)$ were nonsignificantly younger (mean \pm SD age $72 \pm 8.8$ years) than patients reviewed between 12 and 24 months

@ERSpublications

For patients with IPF, length of time in healthcare systems prior to review in an ILD clinic reflects disease severity and may impact upon patient outcome https://bit.ly/2TkO26r

Cite this article as: Brereton CJ, Wallis T, Casey $\mathrm{M}$, et al. Time taken from primary care referral to a specialist centre diagnosis of idiopathic pulmonary fibrosis: an opportunity to improve patient outcomes? ERJ Open Res 2020; 6: 00120-2020 [https://doi.org/10.1183/23120541.00120-2020]. 
$(n=41)(73.5 \pm 7.39$ years $)$ or after 24 months $(n=63)(73.3 \pm 6.6$ years). Patients reviewed in the ILD clinic within 12 months of primary care referral had a significantly preserved mean forced vital capacity (FVC) $(83 \pm 22 \%$ predicted) compared to those reviewed between 12 and 24 months $(75 \pm 22 \%, \mathrm{p}=0.036)$, and $>24$ months $(71 \pm 17 \%, p \leqslant 0.001)$, respectively (figure 1a). There was a trend to decreasing transfer capacity of the lung for carbon monoxide $\left(T_{\mathrm{LCO}}\right)$ with longer time to ILD clinic review; $49.7 \pm 16.7 \%$ predicted for $<12$ months versus $48.0 \pm 20.95 \%$ for $12-24$ months and $45.6 \pm 13.3 \%$ for $>24$ months $(\mathrm{p}=0.089,<12$ versus $>24$ months).

$165(67 \%)$ patients commenced antifibrotic therapy prior to the census date. Kaplan-Meier analysis identified that patients seen in the ILD clinic within 12 months from the initial primary care referral had significantly longer time to discontinuation of therapy compared to those seen at 12-24 or >24 months. The median (95\% CI) time to discontinuation was 774 (508-1040) versus 531 (193-869) versus 390 (247-533) days, respectively, $\mathrm{p}=0.005$ (figure 1b). Multivariate Cox regression analysis, including age at diagnosis, sex, smoking status, FVC \% predicted, $T_{\mathrm{LCO}} \%$ predicted and time to ILD clinic, identified a trend towards the time of first ILD clinic review being an independent predictor of time to discontinuation of antifibrotic therapy (12-24 months, HR 1.63 (95\% CI 0.90-2.92, p=0.104), and >24 months, HR 1.50 (95\% CI 0.95-2.51, p=0.077)), while age (HR 1.04, 95\% CI 1.01-1.07; $\mathrm{p}=0.005)$ and $\mathrm{T}_{\mathrm{LCO}} \%$ predicted (HR $0.98,95 \%$ CI $0.96-1.00 ; \mathrm{p}=0.011)$ were significant independent predictors of time to antifibrotic discontinuation.

116 patients (47\%) were deceased at the time of the census. Patients seen in the ILD specialist clinic within 12 months of primary care referral had significantly longer time to death compared to those reviewed at 12-24 and $>24$ months, with median survival 1558 days (95\% CI 1217-1898 days) versus 883 days (95\% CI 551-1215 days) versus 1063 days (95\% CI 608-1518 days), respectively ( $\mathrm{p}=0.022$ ) (figure 1c). In multivariate regression analysis (age, smoking status, FVC \% predicted, $T_{\mathrm{LCO}} \%$ predicted to time to ILD clinic), time to ILD specialist review was not a significant independent predictor for mortality while age (HR 1.04, 95\% CI 1.02-1.07; $\mathrm{p}=0.001$ ) and $T_{\mathrm{LCO}} \%$ predicted (HR 0.95, 95\% CI 0.94-0.97; $\mathrm{p}<0.001$ ) were significant independent predictors of mortality.

Research into IPF is typically undertaken in specialist centres, with a patient receiving a diagnosis of IPF at this point and the time taken in the healthcare system prior to this point often not available or recorded. Whilst primary care case finding strategies have been advocated, our findings identify that following primary care physician referral, there can be significant variation in referral to a specialist centre. Consistent with our findings, a recent review of Medicare patients identified that one-third of patients visited a respiratory physician more than 3 years prior to an IPF diagnosis [7].

Prior to the approval of antifibrotic therapies, LAMAs et al. [8] identified that a longer delay from the onset of dyspnoea until evaluation at a tertiary care centre was associated with a higher rate of death from IPF independent of disease severity. We identified that patients with IPF who were reviewed in the ILD clinic within 12 months of primary care referral, compared to those seen later, had significantly preserved lung function, significantly longer total duration of antifibrotic therapy and significantly longer time to death.

Our study has a number of limitations. It is a two-centre retrospective study and includes the period following the approval of antifibrotics, although findings were consistent across two distinct primary care healthcare-based systems. Further investigation is required to understand the identified heterogeneity in
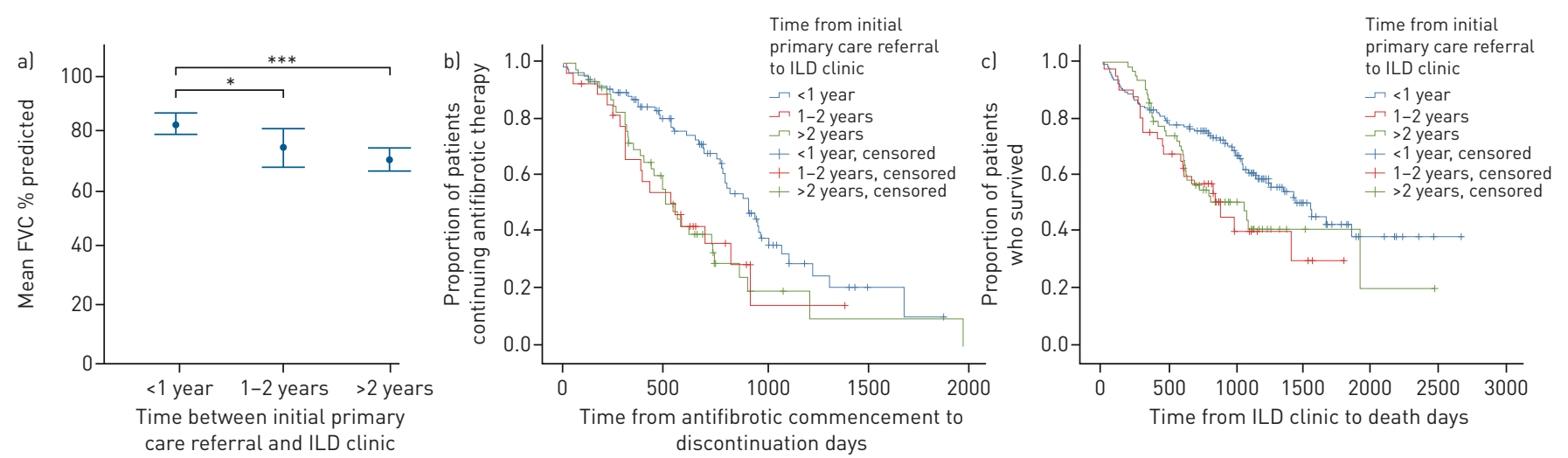

FIGURE 1 Patients diagnosed with idiopathic pulmonary fibrosis stratified by time from first primary care referral to first review within an interstitial lung disease (ILD) clinic. a) Error-bar chart showing the mean forced vital capacity (FVC) \% predicted (error bars represent $95 \%$ confidence intervals). b) Kaplan-Meier analysis of antifibrotic duration and c) Kaplan-Meier analysis of survival. *: $p<0.05 ;{ }^{* * *}$ : $p<0.001$. 
the patient pathway, with potential factors including resource limitations, alternative diagnoses or a period of observation prior to referral, and within the UK cohort, lung function outside of National Institute for Health and Care Excellence criteria for antifibrotic prescribing (FVC 50-80\% predicted).

While the use of cancer service quality measures for the time within cancer pathways is well established to improve patient outcomes [9], no such clinical benchmarks are routine for patients with fibrotic lung diseases. Our findings identify a need for multinational prospective studies to investigate whether the introduction of such standards of care for the time taken within lung fibrosis diagnostic and treatment pathways could improve patient outcomes.

Christopher J. Brereton $\oplus^{1,2}$, Timothy Wallis ${ }^{1}$, Michelle Casey ${ }^{3}$, Lynn Fox ${ }^{3}$, Katarina Pontopiddan ${ }^{1}$, Diane Laws ${ }^{4}$, Jennifer Graves ${ }^{5}$, Vanessa Titmuss ${ }^{6}$, Sarah Kearney ${ }^{7}$, Sian Evans, Alison Grove ${ }^{8}$, Samreen Hamid ${ }^{10}$, Luca Richeldi ${ }^{11}$, Katherine M.A. O'Reilly,12, Sophie V. Fletcher ${ }^{1}$ and Mark G. Jones ${ }^{1,2}$

${ }^{1}$ NIHR Southampton Biomedical Research Centre, University Hospital Southampton, Southampton, UK. ${ }^{2}$ Clinical Experimental Sciences, University of Southampton, Southampton, UK. ${ }^{3}$ Mater Misericordiae University Hospital, Dublin, Republic of Ireland. ${ }^{4}$ The Royal Bournemouth and Christchurch Hospitals NHS Foundation Trust, Bournemouth, UK. ${ }^{5}$ Dorset County Hospital NHS Foundation Trust, Dorchester, UK. ${ }^{6}$ Portsmouth Hospitals NHS Trust, Portsmouth, UK. ${ }^{7}$ Isle of Wight NHS Trust, Newport, UK. ${ }^{8}$ Salisbury NHS Foundation Trust, Salisbury, UK. ${ }^{9}$ Hampshire Hospitals NHS Foundation Trust, Winchester, UK. ${ }^{10}$ Poole Hospital NHS Foundation Trust, Poole, UK. ${ }^{11}$ Universita Cattolica del Sacro Cuore, Fondazione Policlinico Universitario Agostino Gemelli IRCCS, Rome, Italy. ${ }^{12}$ School of Medicine, University College Dublin, Dublin, Republic of Ireland.

Correspondence: Christopher J. Brereton, University Hospital Southampton NHS Foundation Trust, Tremona Rd, Southampton SO16 6YD, UK. E-mail: chrisjbrereton@gmail.com

Received: 6 March 2020 | Accepted after revision: 13 May 2020

Conflict of interest: C.J. Brereton reports nonfinancial support from Boehringer Ingelheim outside the submitted work. T. Wallis has nothing to disclose. M. Casey has nothing to disclose. L. Fox reports nonfinancial support from Roche for a study day in Manchester and nonfinancial support from Boehinger Ingelheim to attend ILD INN, outside the submitted work. K. Pontoppidan reports personal fees for conference attendance from Bayer outside the submitted work. D. Laws has nothing to disclose. J. Graves reports personal fees from GSK for an asthma talk to practice nurses and personal fees for a Pfizer-funded smoking cessation webinar, outside the submitted work. V. Titmuss has nothing to disclose. S. Kearney has nothing to disclose. S. Evans has nothing to disclose. A. Grove has nothing to disclose. S. Hamid has nothing to disclose. L. Richeldi reports personal fees from Biogen for consulting activity; grants and personal fees for membership of an advisory board from Roche; personal fees for consulting activity from ImmuneWorks; grants, and personal fees for membership of a steering committee and an advisory board from Boehringer Ingelheim; personal fees for consulting activity from Celgene and Nitto; personal fees for membership of advisory boards from FibroGen and Promedior; and personal fees for consulting activity from Pliant Therapeutics, Asahi Kasei, Toray, BMS, RespiVant and CSL Behring, all outside the submitted work. K.M.A. O'Reilly reports grants and personal fees for an advisory board from Boehringer Ingelheim, and personal fees for talks and advisory work from Roche, outside the submitted work. S.V. Fletcher reports personal fees and nonfinancial support from Boehringer Ingelheim and Roche/Intermune, outside the submitted work M.G. Jones has nothing to disclose.

\section{References}

1 Richeldi L, Varone F, Bergna M, et al. Pharmacological management of progressive-fibrosing interstitial lung diseases: a review of the current evidence. Eur Respir Rev 2018; 27: 180074.

2 Hoyer N, Prior TS, Bendstrup E, et al. Risk factors for diagnostic delay in idiopathic pulmonary fibrosis. Respir Res 2019; 20: 103 .

3 Maher TM, Strek ME. Antifibrotic therapy for idiopathic pulmonary fibrosis: time to treat. Respir Res 2019; 20: 205.

4 Valeyre D. Towards a better diagnosis of idiopathic pulmonary fibrosis. Eur Respir Rev 2011; 20: 108-113.

5 Purokivi M, Hodgson U, Myllärniemi M, et al. Are physicians in primary health care able to recognize pulmonary fibrosis? Eur Clin Respir J 2017; 4: 1290339.

6 Maher TM, Swigris JJ, Kreuter M, et al. Identifying barriers to idiopathic pulmonary fibrosis treatment: a survey of patient and physician views. Respiration 2018; 96: 514-524.

7 Mooney J, Chang E, Lalla D, et al. Potential delays in diagnosis of idiopathic pulmonary fibrosis in Medicare beneficiaries. Ann Am Thorac Soc 2019; 16: 393-396.

8 Lamas DJ, Kawut SM, Bagiella E, et al. Delayed access and survival in idiopathic pulmonary fibrosis: a cohort study. Am J Respir Crit Care Med 2011; 184: 842-847.

9 National Cancer Intelligence Network, Public Health England. www.ncin.org.uk/cancer_information tools/profiles/ serviceprofiles Date last accessed 18 October 2019 